\title{
Creature:Interactions: A Social Mixed-Reality Playspace
}

\section{Andrew Bluff}

Creativity \& Cognition Studios

University of Technology Sydney

15 Broadway

Ultimo NSW 2007, Australia

Andrew.Bluff@uts.edu.au

Andrew Johnston

Creativity \& Cognition Studios/Animal

Logic Academy

University of Technology Sydney

15 Broadway

Ultimo NSW 2007, Australia

Andrew.Johnston@uts.edu.au

See <www.mitpressjournals.org/toc/leon/50/4> for supplemental files associated with this issue.

\section{Andrew Bluff and Andrew Johnston}

\begin{abstract}
A B S T R A C T
This paper discusses Creature:Interactions (2015), a large-scale mixed-reality artwork created by the authors that incorporates immersive $360^{\circ}$ stereoscopic visuals, interactive technology, and live actor facilitation. The work uses physical simulations to promote an expressive full-bodied interaction as children explore the landscapes and creatures of Ethel C. Pedley's ecologically focused children's novel, Dot and the Kangaroo. The immersive visuals provide a social playspace for up to 90 people and have produced "phantom" sensations of temperature and touch in certain participants.
\end{abstract}

Creature:Interactions (2015) is a large-scale mixed-reality artwork that incorporates immersive visuals, interactive technology, and live actor facilitation to promote full-body movement and social play. The artwork is set in an animated Australian bush landscape that is magically conjured to life by the full-bodied movements of the participants. Participants interact with a range of native Australian animals including koalas, kangaroos, wombats, birds, and lizards represented as giant line-drawn "totem" creatures (Figure I) and photographic particle clouds that morph and dissolve in response to movement. It features a full $360^{\circ}$ interactive visual display that can be presented in $2 \mathrm{D}$ or stereoscopic $3 \mathrm{D}$ and paired with multichannel audio to create a highly immersive experience that can be enjoyed by audiences of up to 90 people simultaneously. The work transports the audience to a number of Outback locations and simulates environmental events such as bushfires and rainstorms before ascending to the virtual treetops to interact with the moon and night stars.

The work that we created is an interactive companion piece to the Creature:Dot and the Kangaroo [I] physical theater show inspired by Ethel C. Pedley's classic Australian children's novel [2] in which a little girl, Dot, is lost in the bush and befriends a mother kangaroo. The kangaroo gives Dot some magical "berries of understanding" that give her the Doolittlian ability to talk with the animals, and she embarks on an ecologically driven journey to understand humanity's negative impact on the natural environment. The interactive installation allows the audience to interact with the creatures and inhabit the digital world presented in the theater show. The experience begins without sound or projections, and as the audience traverses the seemingly empty space, digital particles are created from their movements, and the virtual bushland slowly emerges from the interactive floating particles. The magical shift from a disappointingly empty space to a fully fledged natural landscape through embodied interaction represents the eating of the berries and puts the audience into a state of "understanding" where they can begin their own transformative journey. Mirroring the surreal juxtaposition of real human participants with the surrounding virtual environment, the oversized interactive animals appear to possess a magical presence, simultaneously at one with their animated environment while exuding an almost-alien presence. The embodied interaction with these creatures physically echoes the relationship that human beings have with their own natural environment. 


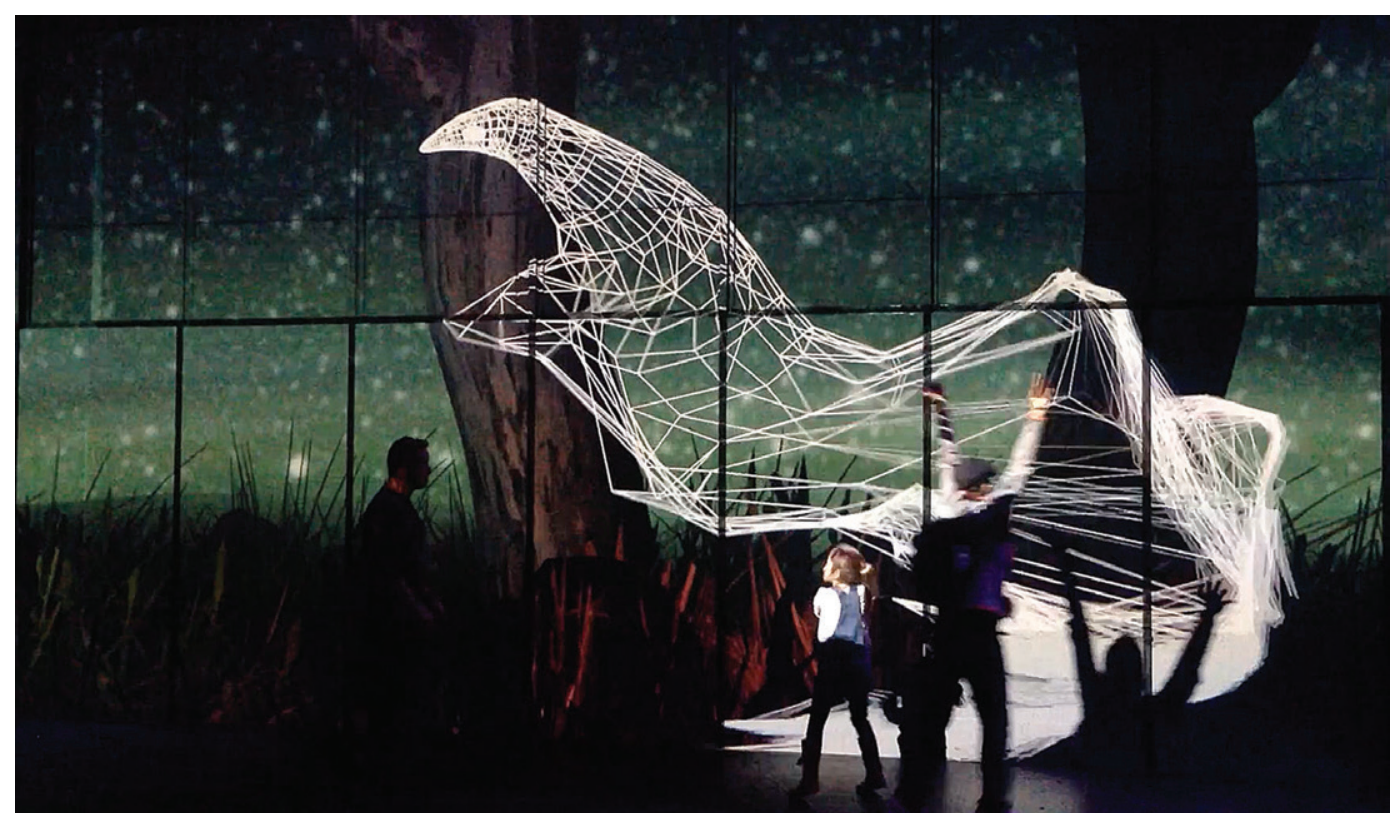

Figure 1. Participants can manipulate giant bird "totems" with their movement in Creature:Interactions. (๑ Stalker Theatre. Photo: Andrew Bluff.)

\section{The Aesthetics of Interaction}

The way that it interacts with the audience is a crucial part of its essence. The core of the art is in the work's behavior more than in any other aspect. —Ernest Edmonds, on interactive art [3]

As its title suggests, Creature:Interactions is in essence an interactive artwork. The work features an embodied interaction between the human and the virtual. It also promotes social interaction among the participants as they try to make sense of this mysterious digital world. As a shared mixed-reality playspace, both types of interactions have equal importance, and the "creature" in the title refers to both the virtual interactive characters and the animalistic, social human participants.

Seminal interactive works with a focus on expressive audio-visual interaction include David Rokeby's Very Nervous System [4], which translated the movements of a single performer into complex and nuanced sounds. The audio-visual performance work Messa di Voce [5] blended vocals and the movement of performers into physics-based projected visuals. The physical nature of the interaction and the generated graphics enabled the creation of rich and complex outputs while keeping the links between performer actions and computer response clear and unambiguous.

More recent works, including Creature:Interactions, the similarly themed Story of the Forest by teamLabs [6], and Connected Worlds by Design I/O [7], feature large immersive environments that allow interactions with large numbers of people simultaneously. In the past, interactive works tended, at least partly for technical reasons, to focus on interaction with only one or two people at a time. The larger size and greater capacity of these recent works have increased the potential for social interactions. We see the increased social aspect as a major opportunity (and also a challenge) for works of this kind.

\section{Digital Interactions}

We designed the digital system in Creature:Interactions to allow a large freedom of movement and embodied expression from the audience. The focus on free-form expressive movement largely stems from the work's origins in physical theater. The system was originally developed for Stalker Theatre's 2012 production Encoded [8] and has since evolved to facilitate the immersive 
Creature:Interactions installation and its associated physical theater show, Creature:Dot and the Kangaroo. Physical theater artists often explore a wide range of movement including handstands, somersaults, and backflips, often using equipment such as stilts, catapults, and trapeze-like slings at great heights (Figure 2). To capture this wide range of free-form movement, a robust infrared motion-tracking system was developed using optical flow algorithms on a network of cameras. The system picks up any movement and allows the artwork to be used by individuals or crowds. Free from any prescribed movement, the participants have been observed waving, jumping, dancing, kicking, cartwheeling, and performing free-form tai chi as they explore their embodied relationship with the surreal virtual world.

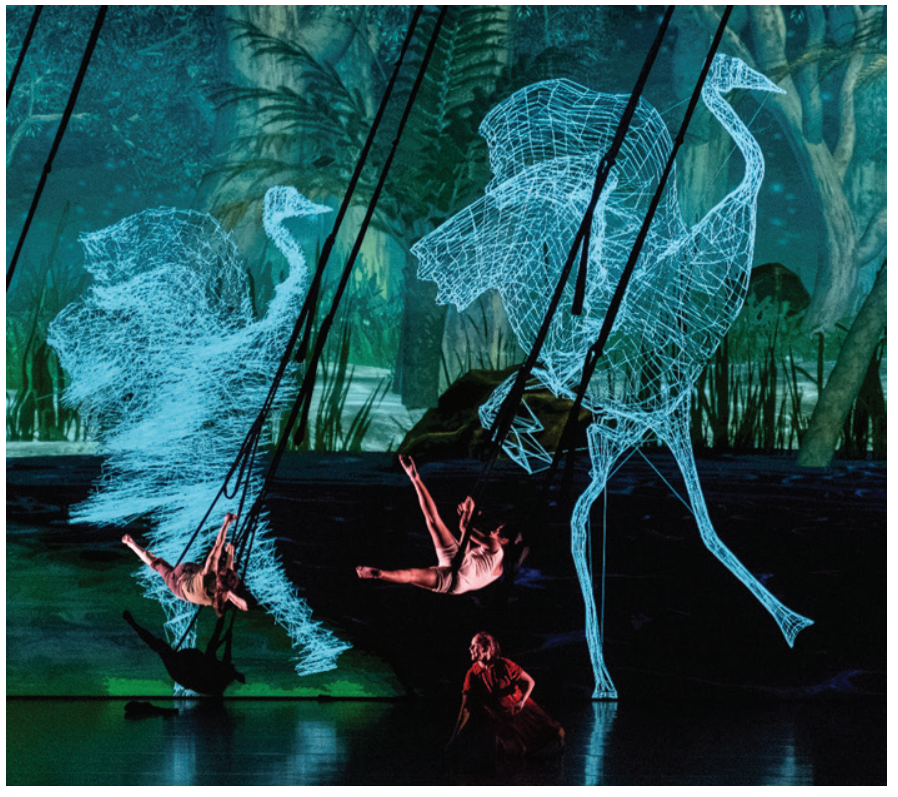

Figure 2. Performers flying through the air on slings in the companion theater show, Creature:Dot and the Kangaroo. (๑ Stalker Theatre. Photo: Darren Thomas.)
To provide the artwork with nuanced and expressive reactions to these free-form movements, the interaction aesthetic was driven by real-world physics simulations. In our design we favored "continuous" gestural interaction where all movements of the participants result in the exertion of virtual forces on simulated physical objects in the virtual world. The intention is that this continuous, physical interaction leads to visual responses that are both complex and intuitively understandable, providing a rich scope for creative expression and discovery. In the field of musical interaction design, the

expressive potential of continuous (as opposed to discrete) interaction paradigms and complex, physics-based interactions have been well explored by Hunt [9], Momeni \& Henry [ı]], Wessel $\&$ Wright [II], and Cadoz et al. [I2]. We draw on these ideas to promote a creative and expressive experience in the visual rather than sonic domain.

The system employs two separate physical systems—a fluid simulation and a rigid-body collision system. The fluid simulation allows star-like particles to gracefully float about the digital night sky in response to audience movements. Movement detected by the camera is fed into a simulation of fluid dynamics, and as the participants move they effectively "stir" this virtual fluid. The particles float on top of this virtual fluid and gracefully flow about the space. Similarly, a multitude of moon balls (3D spheres textured to resemble a full moon) use a rigidbody collision system that lets the objects bang against one another realistically as the audience throws them around in $3 \mathrm{D}$ space. The fluid simulation and collision system allow the stars and moons to fly about the space, forming abstract patterns in response to the free-form movement of the audience, creating a more expressive and "conversational" [I3] style of interaction where both system and participants are reacting to each other.

To theme the system for the Australian Outback, an attraction system was created to force more literal shapes from the more abstract particle systems. The particles are attracted to the shape of 
animal models and rendered as lines to form the giant totemistic animal models that float and warp as the participants stir the virtual fluid. While the line-drawn totems return to their animalistic shapes in the absence of movement, the photographic particle cloud creatures (Figure 3) only appear when movement is detected, disappearing into the virtual ether when stillness prevails. Animated models of birds and butterflies are controlled by the same collision system as the moon balls, but have an added attraction system that flocks around the audience as they move about the space.

The free-form motion tracking, physical simulations, and attraction systems allow a complex but understandable response to movement. The warping of totem creatures, revealing of photographic animals, flocking of birds, throwing of moons, and floating of stars give the audience a range of interaction aesthetics that can be explored through full-bodied, freeform interaction.

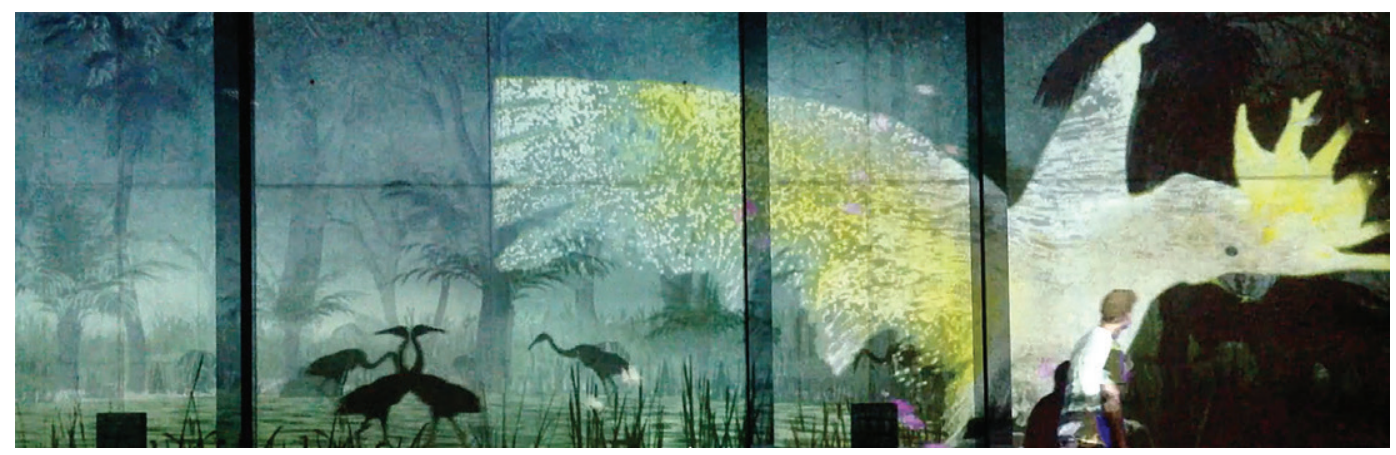

Figure 3. Photographic particle clouds emerge from movement in Creature:Interactions. (๑ Stalker Theatre. Photo: Andrew Bluff.)

\section{Human Interactions}

In a social mixed-reality environment, the way humans relate with the digital is only half of the story. The participants have a shared experience as they explore the digital environment and often form small groups to throw moon balls at each other or warp the creatures in unique ways. Live facilitators engage with children to suggest different forms of movement as they interact with the system and each other. The facilitators suggest the children "move like a creature from the Australian bush" to expand their palette of movement beyond simple hand-waving. The children hop like kangaroos, flap their arms like kookaburra, jump like frogs, and slither like snakes, which changes the interaction aesthetic and allows them to further connect with the virtual bush environment.

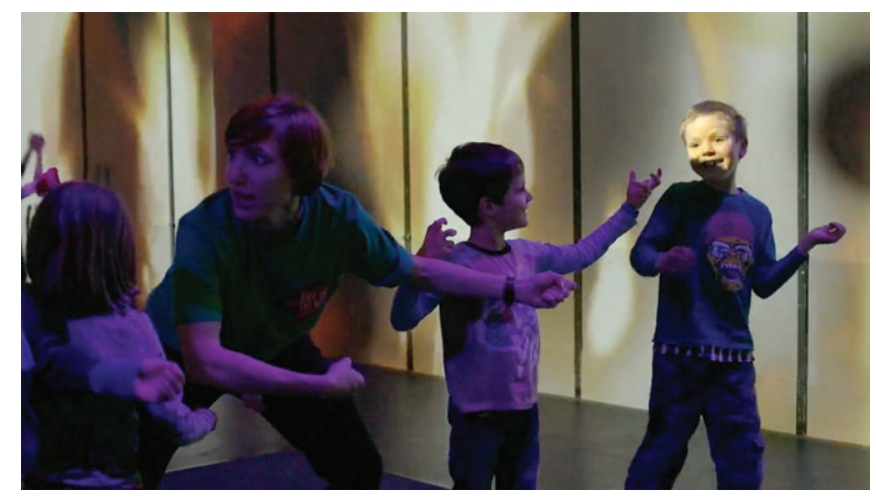

Figure 4. A facilitator working in a group to extinguish the virtual bush fire in Creature:Interactions, 2015. (๑ Stalker Theatre. Video still: Jaina Kalifa.)
The facilitators also form minigroups with the children to work together on tasks such as putting out a bushfire with imaginary buckets of water and hoses (Figure 4), or jumping like frogs in front of the "totem" creatures. The interactive system responds better to combined movement, so grouping together is an effective way to push the graphic response further than what is possible with just one person. As 
groups of children work together to move the reactive visuals, they can observe, copy, and improve upon movements that others are using. It is common to hear children excitedly say to each other, "Check out what I just did! Do you want to try it with me?" Many of the children visiting the artwork don't know each other, and the shared experience of embodied exploration has proven to be a powerful icebreaker. When surveyed about the artwork, one of the facilitators responded, "The most interesting interactions were actually amongst kids that didn't know each other working together to move and create shapes with the animals."

Adults also naturally form social groups when experiencing the work. Many adults were observed working together to tie knots in the virtual totem graphics, interacting at either end of a photographic particle creature to reveal the entire image or playing a sort of ad hoc volleyball game with the floating moon balls. Whether the audience members know each other or not, the piece is a shared experience that creates a unique bond between the participants as they figure out how their movements affect the virtual world.

\section{A Shared Immersion}

The system running Creature:Interactions is scalable, allowing the interactive artwork to be displayed in three different formats. The first features a single wall of interactive $2 \mathrm{D}$ projections, while the second uses a continuous $360^{\circ}$ display projected onto four walls to create an immersive shared experience. Six infrared motion-tracking cameras, multiple computers, and six highdefinition projectors were networked together to create a seamless projection canvas up to five meters high on all four walls and a large interaction zone covering the $18 \mathrm{~m} \times \mathrm{I} 2 \mathrm{~m}$ space. The third type of installation uses the same $360^{\circ}$ canvas, but renders all of the visuals in stereoscopic $3 \mathrm{D}$ to provide an even more immersive mixed-reality experience. Members of the audience wear stereoscopic $3 \mathrm{D}$ glasses, which expands the depth of the bush landscape and allows the creatures, particles, and moon balls to jump off the walls and float anywhere within the shared space.

While the installation on one single wall is enjoyable, the shift to a $360^{\circ}$, four-wall display represents a surprisingly large jump in terms of immersive effect. Following the two-week season at the Queensland Performing Arts Centre, the artists, directors, and facilitators of the piece were all interviewed to explain how they approached, designed, and received the interactive artwork. All interviewees, alongside many members of the audience, commented on the significant extent to which the $360^{\circ}$ display improved the piece.

I think there's something to be said about it being 36o. I think it's just kind of striking on the body and the brain. ...It seems to suit the thing better, you are inside a digital environment, you are inside an animated environment. —Director, Creature:Interactions

The large $360^{\circ}$ projections were described as "striking," "aesthetically interesting," and "overwhelming," and every single person used the term "immersive" to describe it. Many people observed that the surrounding nature of the system ensured that everyone was interacting with it. Unlike single-wall systems, the $360^{\circ}$ setup ensures that no matter where you turn or where you step, you cannot help but participate and interact with the work-there is no escaping it.

Perhaps most importantly, the impressive nature of the interactive visuals actually invited the audience to engage and interact with it.

The scale of it was incredibly impressive, ... when it first came up ... it was all the way around them. You could just see the kids going, "Wow. I want to play with this. I want to explore it." —Lead facilitator, Creature:Interactions 


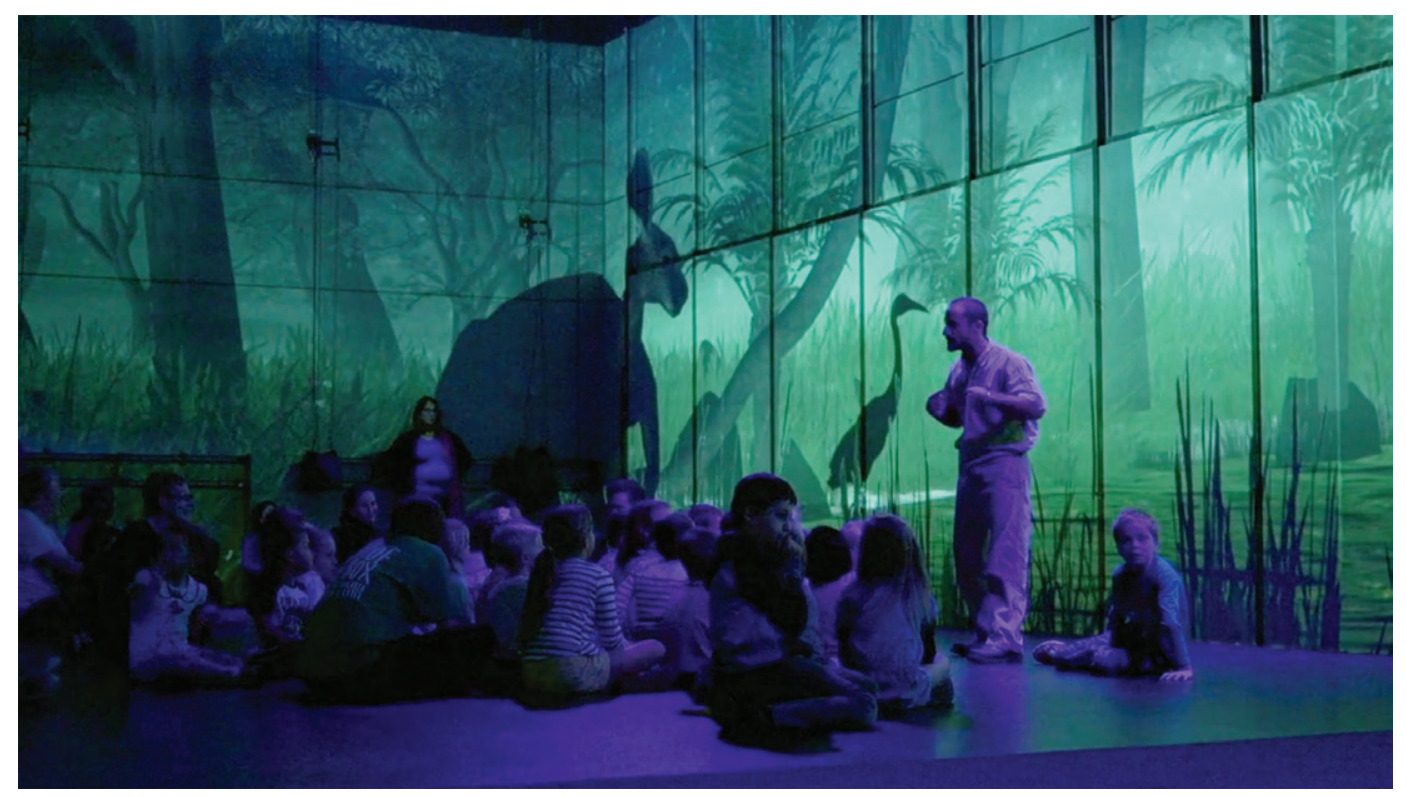

Figure 5. Facilitator briefs the children inside the large $360^{\circ}$ Creature:Interactions playspace at the Queensland Performing Arts Centre. (๑ Stalker Theatre. Video still: Jaina Kalifa.)

\section{Stereoscopic 3D}

The stereoscopic $3 \mathrm{D}$ version of Creature:Interactions has been presented in the University of Technology Sydney's Data Arena, a CAVE-like [I4] facility featuring a cylindrical screen. The Creature software was updated with a custom-built "omnistereo" rendering system to ensure that every angle of the cylindrical screen was warp-free and displayed a seamless $3 \mathrm{D}$ image no matter where the audience stood [I5]. A depth-aware compositing engine was developed to allow the interactive $3 \mathrm{D}$ particles to be rendered inside the prerendered $3 \mathrm{D}$ bush landscape. Interactive butterflies and birds weave in and out of trees, rocks, and grass, passing in front of or behind these prerendered elements as expected. A 3 D tracking system was used to detect motion through the entire space, allowing the participants to throw virtual moon balls into and across the installation space for the night sky finale. A I4-channel sound system was used to attach sounds to virtual $3 \mathrm{D}$ animals as they flocked through and around the space.

Participants who experience both versions consistently remark on the added immersion of the stereoscopic $3 \mathrm{D}$ system, as it moves the interactive elements off the screen and into the space, around their bodies.

\section{The Phantom Limb Experience}

The artwork features a large bushfire scenario that is eventually extinguished by a virtual rainstorm (Figure 6). During the $360^{\circ} 2 \mathrm{D}$ installation, members of the audience reported that they could "feel" the temperature drop during the virtual rain sequence, even though the actual temperature did not change. In addition to this cooling effect, some patrons of the stereoscopic ${ }_{3} \mathrm{D}$ version described a heating effect during the bushfire sequence.

Temperature was not the only phantom sensation activated during the stereoscopic experience. Shortly after the immersive bushscape is conjured into existence at the beginning of the show, one of the participants is given a motion-tracking marker disguised as plastic flowers and told to hold it at arm's length. Controlled by the rigid-body flocking algorithm and rendered seamlessly across all six surrounding projection screens using the omnistereo algorithm, butterflies fly through the space and appear to land on the flowers. When the participant moves, the butterflies scatter and gradually fly back down to the motion-tracking marker held in the outstretched arm. 
Many members of the audience remarked upon the impressive illusion of the $3 \mathrm{D}$ butterflies during this section and were observed discussing the butterflies long after the experience had finished. On two separate occasions, participants have described physically feeling the butterflies touching the ends of their fingers as they appeared to land on their outstretched hands. This phantom touch sensation is yet another example of the brain activating unengaged senses within the immersive digital environment.

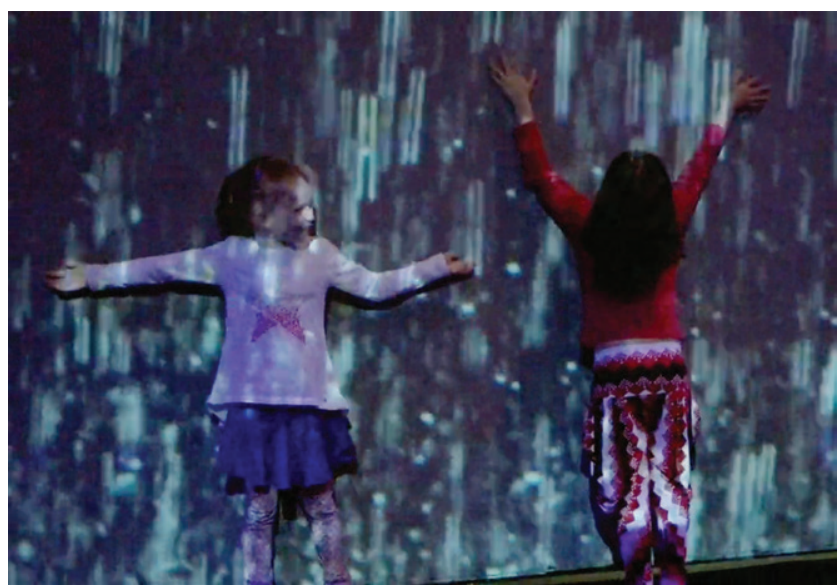

Figure 6. Children playing in digital rain. (๑ Stalker Theatre.

Video still: Jaina Kalifa.)

The phantom temperature and touch effects have never been reported during the one-wall versions. This suggests that the surrounding visuals may trick the brain, empathetically triggering other senses that are known to strongly correlate with the situation at hand. V.S. Ramachandran identified a similar phenomenon in amputees, who report a "phantom" pain or physical sensation in their amputated limb [I6]. He was able to elicit feelings of control and sensations of touch in the phantom limb when patients were able to see the limb move via a mirror reflection of their own remaining limb. The phantom limb effect has been studied extensively in the field of neuroscience, but its application to physical theater and immersive installations provides an interesting direction for future investigation.

\section{The Evolution of Interactions}

The understandable complexity provided by the fluid simulations and rigid-body collision system promoted a rich palette of full-bodied interactions, while the large-scale $360^{\circ}$ display created an immersive and social mixed-reality experience that was further improved by $3 \mathrm{D}$ stereoscopic visuals in the Data Arena. We are currently investigating portable dome projection systems to bring the immersive playspace to remote locations, spreading its environmental message. Creature:Interactions is a companion piece to a physical theater show and, while successful, future developments may explore a tighter integration of the two pieces with the physical actors performing inside the immersive space to create an interactive physical theater experience. The phantom limb effect will also be explored as a method to create empathy in future works.

The combination of physical interaction, immersive technology, and social participation has made Creature:Interactions a unique live experience and heralds a new stream of exploration for the physical theater company.

\section{Acknowledgments}

This work was originally produced by Stalker Theatre and commissioned by Out of the Box Festival. $3 \mathrm{D}$ animation by Boris Morris Bagattini. Thanks to David Clarkson, Benjamin Knapton, Dan Crestani, Tricia Clark-Fookes, and the entire Stalker Theatre team. Research supported by the Australian Government Research Training Program Scholarship. 


\section{References}

I. Stalker Theatre, Creature:Dot and the Kangaroo (2015), <http://www.stalker.com.au/creature>.

2. E. Pedley, Dot and the Kangaroo (New York: HarperCollins Publishers, 20I4).

3. E. Edmonds, "The art of interaction," Digital Creativity 2I, No. 4, 257-264 (2010).

4. D. Rokeby, "The construction of experience: Interface as content" in C. Dodsworth Jr., ed., Digital Illusion: Entertaining the Future with High Technology (Boston: Addision-Wesley Professional, 1998) pp. $27-48$.

5. G. Levin and Z. Lieberman, Messa di Voce (2003), <http://www.flong.com/projects/messa/>.

6. teamLab, Story of the forest (2016), <http://www.team-lab.net/w/story-of-the-forest>.

7. Design I/O, ConnectedWorlds (2015), <http://design-io.com/projects/ ConnectedWorlds>.

8. Stalker Theatre, Encoded (20I5), <http://www.stalker.com.au/encoded>.

9. A. Hunt, M.M. Wanderley, and M. Paradis, "The Importance of Parameter Mapping in Electronic Instrument Design," Journal of New Music Research 32, No. 4, 429-440 (2003).

Io. A. Momeni and C. Henry, "Dynamic Independent Mapping Layers for Concurrent Control of Audio and Video Synthesis," Computer Music Journal 30, No. I, 49-66 (2006).

II. D. Wessel and M. Wright, "Problems and Prospects for Intimate Musical Control of Computers," Computer Music Journal 26, No. 3, II-22 (2002).

I2. C. Cadoz, A. Luciani, and J.L. Florens, "Responsive input devices and sound synthesis by simulation of instrumental mechanisms: The cordis system," Computer Music Journal 8, No. 3, 60-73 (I984).

I3. A. Johnston, "Fluid simulation as full body audio-visual instrument," Proceedings of the conference on New Interfaces for Musical Expressions, p. I32 (2013).

I4. C. Cruz-Neira, D.J. Sandin, and T.A. DeFanti, "Surround-screen projection-based virtual reality: the design and implementation of the cave," Proceedings of the 2oth annual conference on Computer Graphics and Interactive Techniques, pp. I35-I42.

15. A. Simon, R.C. Smith, and R.R. Pawlicki, "Omnistereo for panoramic virtual environment display systems," Virtual Reality, Proceedings, IEEE, pp. 67-279 (2004).

I6. V.S. Ramachandran and W. Hirstein, "The perception of phantom limbs. The DO Hebb lecture," Brain I2I, No. 9, 1603-1630 (1998). 